\title{
Least Action Principle in Physics
}

\author{
Muhammad Gaffar ${ }^{1}$ \\ ${ }^{1}$ Bachelor Student at Department of Physics \\ Universitas Indonesia, Depok 16425
}

17 Nov 2017

\begin{abstract}
We learn physics by starting it from classical problem, where Newton's laws govern the classical physics. In high school or often in early physics in undergraduate, we still solve classic problem with newton's equation of motion. In this article, I will show that there's more alternative way to see classical problem instead seeing from Newton's law, where it seems so boring. The alternative ways we will see that is Lagrangian formula and Hamiltonian's equation of motion. And you will see that Newton's laws less fundamental from alternative methods where they are derived from more fundamental principle, called Least Action Principle. They are still same physics and same result, but different perspective and advantages. Newton's laws have their own advantages, so as Lagrangian and Hamiltonian. Yet, from Lagrangian and Hamiltonian we can see further the beautiful of physics and mathematics inside. Furthermore, we will not just see Least Action Principle in classical paradigm, since we are now lives in physics are governed by Quantum mehcanics, so we will see this principle's completeness from Quantum paradigm. Again, we will see the beautifulness of this principle arise in Quantum Mechanics, and of course the strangeness too.
\end{abstract}

\section{Introduction}

The principle of least action has been great tool for discovery law of nature. Thanks to Euler, Lagrange, Hamilton, and Jacobi that transform this useful principle to mathematical sense. We have tackle many classical problem, like particle trajectory, or whatever with Euler-Lagrange or Hamilton-Jacobi equation. Unfortunately, often as student, appreciation to this method often just limited by how it can solve analytical problems. Sometimes, or even rarely we are lack of how we interpret the mathematics behind this principle. So here, we are asking how are actually the meaning behind this principle and meaning of the its mathematics. In addition, I will show new paradigm of this principle arise in quantum mechanics.

\section{A Boring Method}

Before I dive too deep about least action principle, let me give short explanation about old-method in classical mechanics, newtonian method. Suppose you have particle with position $\vec{r}(t)$, and acted upon by a force $\vec{F}$. By Newton's Laws, we can write down the equation to be

$$
\vec{F}=m \vec{a}
$$

or in more gorgeous way,

$$
m \vec{r}=-\vec{\nabla} V(r)
$$

This second-order differential equation* have two integration constant, initial position $\vec{r}(t)$ and initial velocity $\vec{v}(t)$, where $V(r)$ is potential acted upon on system. And voila!, we can draw the trajectory or prediction about how system behave (usually position $\times$ time). Yet, there's problems in this method. First, it contains vector, where we need consider the relative position, or relative velocity. Second, it just about initial information (position and velocity). Okay, in other persepctive the

\footnotetext{
*In more general form, where Cartesian coordinates are not always convenient. In a set of curvilinear coordinates, Newton's second law is

$$
F^{a}=m\left(\frac{d^{2} \xi^{a}}{d t^{2}}+\Gamma_{b c}^{a} \frac{d \xi^{b}}{d t} \frac{d \xi^{c}}{d t}\right)
$$
}


second problem is not really a problem, but in most case we want solve a problem where we know the initial information, and final information that we want to be. Third, it is hard and takes long time to solve in system contains more object to taken into account. Fourth, it is boring. About 4 years we solve mechanics problem with just one method and limited case (high school to second-semester college). At last but not least, we cannot derive least action principle.

\section{Action in nutshell}

If you read carefully this principle, for first time you must be disturbed by the 'action' notion (at least for me in the first time). So, why least action, not lowest possible energy? Words like 'energy' are bandied about as if we know exactly what we mean (when we do not), and these have even been embodied symbolically in many famous equations of physics. (Perhaps there exist more refined concepts.) Among these old concepts are the familiar definitions of potential energy and kinetic energy. But if, as Einstein discovered, all motion (speed) is relative, and if all positions are relative, it appears that these concepts of 'potential and kinetic energy' need to be, in some sense, qualified ${ }^{6}$. So we need alternative way to look how nature works, especially trajectory or history problems. The standard and most used definition of action was defined by Hamilton,

$$
S \equiv \int_{0}^{t} L(x, \dot{x} ; t) d t
$$

Where $L(x, \dot{x} ; t)$ is Lagrangian,

$$
L \equiv \frac{1}{2} m \dot{x}^{2}-V(x)
$$

Universally, energy must be conserved. In same sense, there must be properties need to be hold in action. Yes, nature works where action must be stationary,

$$
\delta S=0
$$

Wait, what's the meaning behind integral of lagrangian? and where minus sign come from? Well, it may be hard too make action mathematical definition intuitive. But it works, we can derive equation of motion from action mathematical definition where action must be stationary, and widened to scope of Electrodynamics, General Relativity, and Quantum Mechanics. Well, if you still not convinced enough, let me give you some illustration and derivation equation of motion from (3) and (5) after that. This illustration was narrated by Feynman ${ }^{2}$,

Suppose you have a particle (in a gravitational field, for instance) which starts somewhere and moves to some other point by free motionyou throw it, and it goes up and comes down (Fig. 1). It goes from the original place to the final place in a certain amount of time. Now, you try a different motion. Suppose that to get from here to there, it went as shown in Fig. 2 but got there in just the same amount of time. Then he said this: If you calculate the kinetic energy at every moment on the path, take away the potential energy, and integrate it over the time during the whole path, youll find that the number youll get is bigger than that for the actual motion.

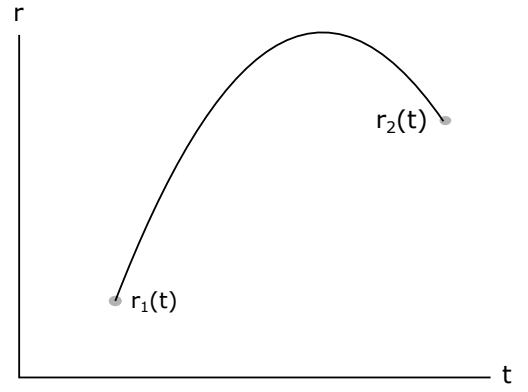

Figure 1.

In other words, the laws of Newton could be stated not in the form $F=m a$ but in the form: the average kinetic energy less the average potential energy is as little as possible for the path of an object going from one point to another. 


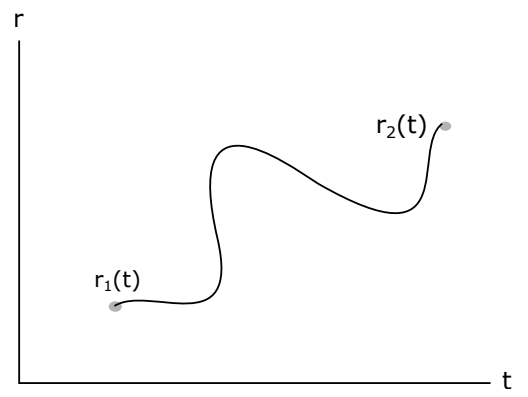

Figure 2.

Has been convinced enough yet? No? Okay as I promised, I'll derive equation ${ }^{1}$ of motion in this mathematical motion. But you might pay attention that action isn't regular function. It is functional (a function of a function). So $\delta S=0$ is not ordinary calculus of maxima and minima, where you calculate the action and just differentiate to find minimum. In fact, it is called calculus variation.

Suppose a given path $\vec{r}(t)$. We ask how the action changes when we change the path slightly from original path

$$
\vec{r}(t) \rightarrow \vec{r}(t)+\delta \vec{r}(t)
$$

Where we need to keep end points of the path fixed

$$
\delta \vec{r}\left(t_{1}\right)=\delta \vec{r}\left(t_{2}\right)=0
$$

So the action-equation (3)-for new path

$$
S(\vec{r}+\delta \vec{r})=\int_{t_{1}}^{t_{2}} d t\left[\frac{1}{2} m\left(\dot{\vec{r}}^{2}+2 \dot{\vec{r}} \cdot \delta \dot{\vec{r}}+\delta \dot{\vec{r}}^{2}\right)-V(\vec{r}+\delta \vec{r})\right]
$$

We expand potential $V$ with taylor expansion

$$
V(\vec{r}+\delta \vec{r})=V(\vec{r})+\vec{\nabla} V \cdot \delta \vec{r}+\ldots
$$

Since $\delta \vec{r}$ is small enough so that second-order and so on can be ignored. Then difference between action in perturbed path and unperturbed path is

$$
\delta S \equiv S(\vec{r}+\delta \vec{r})-S(\vec{r})=\int_{t_{1}}^{t_{2}} d t(m \dot{\vec{r}} \cdot \delta \dot{\vec{r}}-\vec{\nabla} V \cdot \delta \vec{r})
$$

Integration by parts, we get

$$
S(\vec{r}+\delta \vec{r})-S(\vec{r})=\int_{t_{1}}^{t_{2}} d t(-m \ddot{\vec{r}}-\vec{\nabla} V) \cdot \delta \vec{r}+(m \dot{\vec{r}} \cdot \delta \vec{r})_{t_{1}}^{t_{2}}
$$

This picks up a term that is evaluated at the boundaries $t_{1}$ and $t_{2}$. However, this term vanishes since the end points are fixed, eq. (7). Hence, we get

$$
\delta S=\int_{t_{1}}^{t_{2}} d t[-m \ddot{\vec{r}}-\vec{\nabla} V] \cdot \delta \vec{r}
$$

The condition we need that is $\delta S=0$. The only way this can be true if

$$
m \ddot{\vec{r}}=-\vec{\nabla} V
$$

Hey, it is just newton's second law! So for requiring law satisfied, the action must be stationary $\delta S=0$, like we assumed it before. It's astounding concept!

The term 'least' in least action principle may be tricky, because what I proved before is not that action must be minimum, in fact, it's not always minimum. That's why instead using term 'minimum' or 'lowest', I used 'stationary' frequently to state the action must be in stationary. So here, 'least' is first-order of change in the value of $S$, when you change path, is zero. It is not necessarily minimum. 


\section{Sophisticated Method}

\subsection{Euler-Lagrange}

Okay, we have derived the equation of motion. Then what? of course, the goal is not just to show derivation of equation of motion. But, to give alternative way to solve mechanics problem. Instead using initial information, how about initial and final information, i.e position. This is what called Euler-Lagrange equation offers. There's no new physics in Euler-Lagrange equation, just new perspective in understanding nature, where nature works in least action principle. Before, the change value of action $\delta S=0$. So we can derive Euler-Lagrange from the functional of action $S$. After some math*, we get Euler-Lagrange (EL) equation

$$
\frac{\partial L}{\partial q_{j}}-\frac{d}{d t} \frac{\partial L}{\partial \dot{q}_{j}}+\sum_{k} \lambda_{k}(t) \frac{\partial f_{k}}{\partial q_{j}}=0
$$

Where $L=T-V, T$ as kinetic energy of system, and $V$ as potential acted upon by system as I write in equation (4). We can say that $q$ is generalized coordinates. It is therefore worth pointing out that Lagrange's equations expressed above, the undetermined multipliers $\lambda_{k}(t)$ are closely related to the forces of constraint ${ }^{7}$. You see that Lagrangian's method is simpler where we deal only with energy of the system. It's scalar not vector. In fact it is super useful in quantum mechanics where we normally know the energies but not the forces.

Although there's no difference in result of mechanics analysis in body of system. But from philosophical viewpoint, we can make distinction. Let me re-state how nature works in least action principle. In the Newtonian formulation, a certain force on a body produces a definite motion-that is, we always associate a definite effect with certain cause. According to Hamilton's principle, however, the motion of a body results from the attempt of nature achieve a certain purpose, namely, to minimize the time integral of difference between the kinetic and potential energies ${ }^{7}$.

EL equation is really good equation. It follows many rule of physics, well if not, it's not good equation. Let me present you one of the most beautiful and useful theorems in physics. It deals with two fundamental concepts, namely symmeries and conserved quantities. It's called Noether's theorem.

Noether's theorem: For each symmetry of the Lagrangian, there is a conserved quantity.

Through this beautiful theorem, let me prove the some properties of Lagrange Equation.

Consider a small shift of coordinates,

$$
q_{i} \rightarrow q_{i}+\epsilon K_{i}(q)
$$

$K_{i}(q)$ may be a function of all the $q_{i}$. The fact that the lagrangian does not change at first order in $\epsilon$ means that

$$
\begin{aligned}
0=\frac{d L}{d \epsilon} & =\sum_{i}\left(\frac{\partial L}{\partial q_{i}} \frac{\partial q_{i}}{\partial \epsilon}+\frac{\partial L}{\partial \dot{q}_{i}} \frac{\partial \dot{q}_{i}}{\partial \epsilon}\right) \\
& =\sum_{i}\left(\frac{\partial L}{\partial q_{i}} K_{i}+\frac{\partial L}{\partial \dot{q}_{i}} \dot{K}_{i}\right)
\end{aligned}
$$

Let me rewrite in momentum, where $p \equiv \frac{\partial L}{\partial \dot{q}_{i}}$ Then we can write

$$
0=\sum_{i}\left(\dot{p} K_{i}+p \dot{K}_{i}\right)
$$

It's easy to check

$$
0=\frac{d}{d t} \sum_{i} p K_{i}
$$

Hence, we get

$$
\sum_{i} p K_{i}=Q
$$

\footnotetext{
*You can easily check the derivation in your textbook, i.e Marion Thronton or David Morin
} 
for $K_{1}=K_{2}=1$, we see that

$$
Q=p_{1}+p_{2}
$$

This is conservation of linear momentum. and for $K_{1}=q_{2}$ and $K_{2}=-q_{1}$, we see that

$$
Q=q_{2} p_{1}-q_{1} p_{2}=\vec{q} \times \vec{p}
$$

And this is conservation of angular momentum. And let me prove you one last thing where every methods must hold, the conservation of energy. In closed system, the system has time translation symmetry. So,

$$
\frac{d L}{d t}=0
$$

So, total derivative of Lagrangian becomes

$$
\frac{d L}{d t}=\sum_{j} \frac{\partial L}{\partial q_{j}} \dot{q}_{j}+\frac{\partial L}{\partial \dot{q}_{j}} \ddot{q}_{j}
$$

But we know before, the lagrange's equation for zero constraint are

$$
\frac{\partial L}{\partial q_{j}}=\frac{d}{d t} \frac{\partial L}{\partial \dot{q}_{j}}
$$

Subtitute equation (22) to (21),

$$
\frac{d L}{d t}=\sum_{j} \dot{q_{j}} \frac{d}{d t} \frac{\partial L}{\partial \dot{q}_{j}}+\frac{\partial L}{\partial \dot{q}_{j}} \ddot{q}_{j}
$$

We can easily check that

$$
\frac{d L}{d t}-\sum_{j} \frac{d}{d t}\left(\dot{q}_{j} \frac{\partial L}{\partial \dot{q_{j}}}\right)=0
$$

so that

$$
\frac{d}{d t}\left(L-\sum_{j} \dot{q_{j}} \frac{\partial L}{\partial \dot{q}_{j}}\right)=0
$$

We denote the constant by $-H$

$$
L-\sum_{j} \dot{q}_{j} \frac{\partial L}{\partial \dot{q_{j}}}=-H
$$

Where

$$
\frac{\partial L}{\partial \dot{q}_{j}}=\frac{\partial(T-V)}{\partial \dot{q}_{j}}=\frac{\partial T}{\partial \dot{q}_{j}}
$$

Then, equation (26) can be written as

$$
T-V-\sum_{j} \dot{q_{j}} \frac{\partial T}{\partial \dot{q_{j}}}=-H
$$

You can check by yourself ${ }^{7}$ that $\sum_{j} \dot{q}_{j} \frac{\partial T}{\partial \dot{q}_{j}}=2 T$, so

$$
T-V-2 T=-H \Rightarrow T+V=H
$$

The total energy is a constant of the motion for this case. The function $H$, called Hamiltonian of the system. We can see that it's beautiful that simple principle of action sum up the fundamental conservation law.

As I said before, Today, we use the Lagrangian method to describe all of physics, not just mechanics. All fundamental laws of physics can be expressed in terms of a least action principle. This is true for electromagnetism, special and general relativity, particle physics, and even more speculative pursuits that go beyond known laws of physics such as string theory ${ }^{1}$. For example, (nearly) every experiment ever performed can be explained by the Lagrangian of the Standard Model

$$
L \sim R-\frac{1}{4} F_{\mu \nu} F^{\mu \nu}+i \bar{\psi} \gamma^{\mu} D_{\mu} \psi+\left|D_{\mu} h\right|^{2}-V(|H|)+h \bar{\psi} \psi
$$

Dont worry if you dont understand many of the symbols. You are not supposed to. View this equation like art. 


\subsection{Hamiltonian Equation of Motion}

After Hamilton formulated his Hamilton's principle, the Least Action Principle, unfortunately, Lagrange derived the alternative method of newton's equation of motion first using his principle. Ironic. Although he was preceded, he derived new equation of motion. Same physics, and same result, but different approach. Instead using function $L(q, \dot{q} ; t)$, we will using new function $H(q, p ; t)$. Before that, we define generalized momentum* as $p_{j}$

$$
p_{j} \equiv \frac{d L}{d \dot{q}_{j}}
$$

In equation (26), we have find that Hamiltonian may be written

$$
H(q, p ; t)=\sum_{j} p_{j} \dot{q}_{j}-L(q, \dot{q}, t)
$$

Then we have

$$
\delta H=\sum\left(\delta \dot{q}_{i} p_{i}+\dot{q}_{i} \delta p_{i}-\frac{\delta L}{\delta \dot{q}_{i}} \delta \dot{q}_{i}-\frac{d L}{\delta q_{i}} \delta q_{i}\right)-\frac{d L}{d t}
$$

The first and third terms in the bracket cancel, because we have defined generalized momentum in equation (31). Then we have

$$
\delta H=\sum\left(\dot{q}_{i} \delta p_{i}-\frac{d L}{\delta q_{i}} \delta q_{i}\right)-\frac{d L}{d t}
$$

Furthermore, In lagrange equation we can write

$$
\dot{p}_{j}=\frac{d L}{d q_{j}}
$$

Hence, equation (34) becomes

$$
\delta H=\sum_{i}\left(\dot{q}_{i} \delta p_{i}-\dot{p}_{i} \delta q_{i}\right)-\frac{d L}{d t}
$$

Hey, but we know that $H$, solely as a function of $q_{i}$ and $p_{i}$, with no explicit dependence on the $\dot{q}_{i}$, in other words ${ }^{3}$ as I wrote in eq. (32) where $H(q, p ; t)$

$$
\delta H(q, p ; t)=\sum_{i}\left(\frac{\partial H}{\partial p_{i}} \delta p_{i}+\frac{\partial H}{\partial q_{i}} \delta q_{i}\right)+\frac{d H}{d t}
$$

As comparison by equation of (34) we have

$$
\dot{q}_{j}=\frac{\partial H}{\partial p_{i}} \quad \text { and } \quad \dot{p}_{j}=-\frac{\partial H}{\partial q_{i}}
$$

and as addition

$$
\frac{d H}{d t}=-\frac{d L}{d t}
$$

Eq. (38) and (39) is exactly the Hamiltonian's Equation of Motion!

But what is the meaning of Hamiltonian? as we seen previously, that Hamiltonian equals the total energy $T+V$. Under condition ${ }^{7} \frac{\partial H}{\partial t}=0$, then $H=E=$ constant. Hamiltonian so useful in quantum mechanics, and part of Schrodinger's equation.

Often for complicated dynamical system, we usually represent both positions and momentum of all particles, this is called Hamiltonian phase space. Hamiltonian formulation of dynamics is ideal for the statistical study of complex systems, thus we may consider the representative points to be sufficiently numerous that we can define a density in phase space $\rho$. From hamiltonian dynamics, we can prove one of the fundamental theorem, called Liouville's Theorem, and see that

$$
\frac{d \rho}{d t}=0
$$

It states that the density of representative points in phase space corresponding to the motion of the system of particle remains constant during the motion. Thus we must use Hamiltonian dynamics rather than Lagrangian dynamics to discuss ensembles in statistical mechanics.

\footnotetext{
*Although it is defined, it's not arbitary definition, it's real momentum in the system
} 

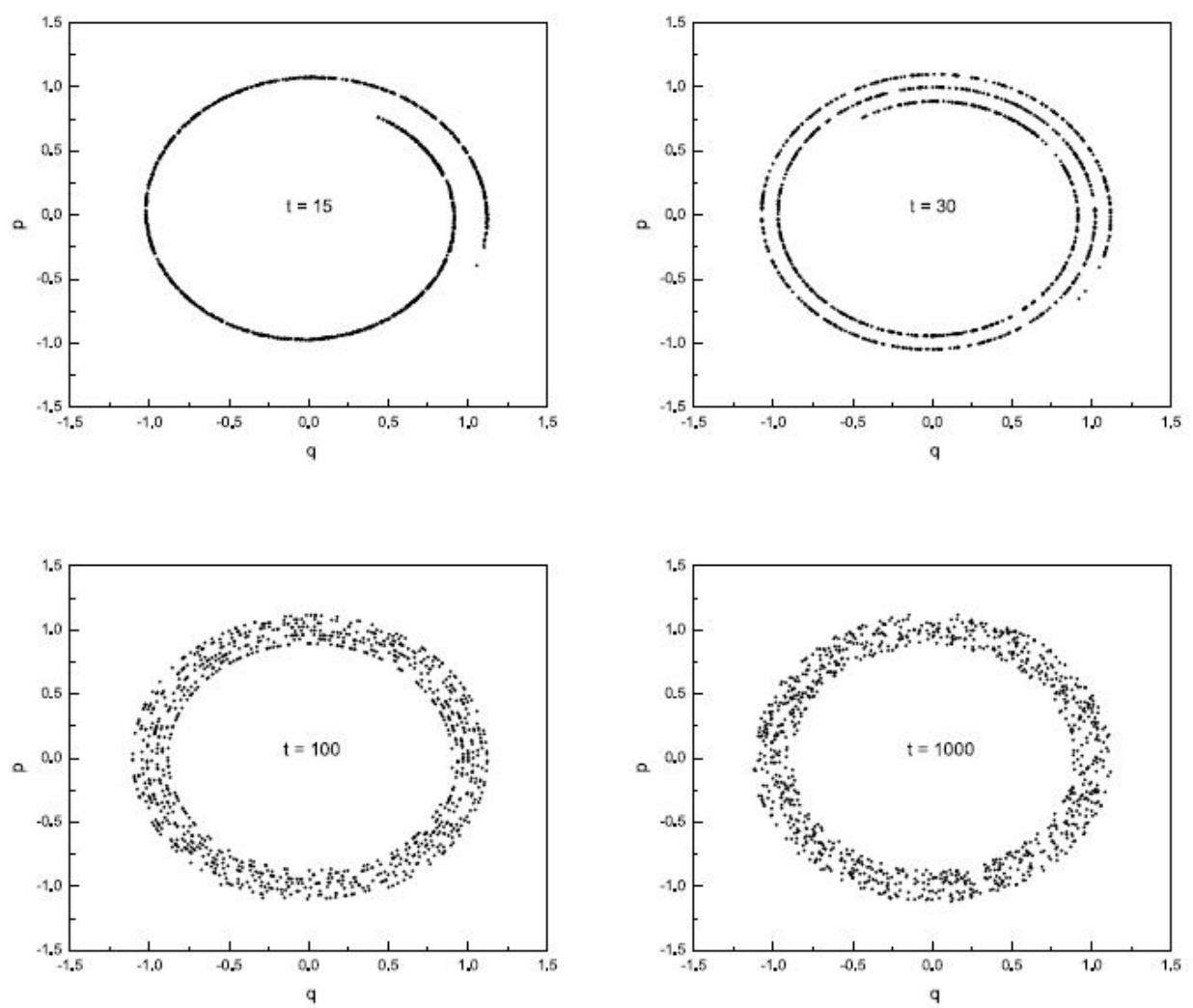

Figure 3. Evolution of the ensemble of 1000 systems described by the Hamiltonian ${ }^{5}$

In some aspect, we can say that Hamiltonian is better than Lagrangian, also we can say it reversely. In Hamiltonian we have Hamiltonian phase space and Liouville's theorem, where it represents the time evolution dynamics directly. It seems Lagrangian sound less useful for time evolution. On other hand, Lagrangian actually gives more insight into the symmetries of the system, the Noether's Theorem.

\section{Quantum Is Strange}

Previously, we have discussed dynamical of system in classical, and it seems so intuitive and common sense. I hate to say this, that what i told you before, that nature choose path where the action is stationary, is a lie. Don't be pessimistic quickly. It's not completely lie, it just our statement is incomplete. Got You!

There's something less intuitive in least action principle. I should discuss this in section "Action In Nutshell", but i suspend it for this section. In Newton's Law, system moves under the law itself, or we can say it "cause and effect". But something wrong when I gave you statement that system choose path where action is stationary. How can system know it the path is stationary? what a decision making! How system evaluate other path? See? something not going right here, as i said, incomplete.

In Quantum Mechanics, particle can behave like wave. So there's properties like amplitude, phase, and superposition. Particle actually choose all paths! It do not know where the least is. But if we see particle as wave, It goes to all direction! Of course that's not what we observe. When wave went on a path that took different amount of times, it would arrive at different phase. The total amplitude at some point is superposition of all different ways the light can arrive. All the paths that give wildly different phases dont add up to anything. But if you can find a whole sequence of paths which have phases almost all the same, then the little contributions will add up and you get a reasonable total amplitude to arrive. The important path becomes the one for which there are many nearby paths which give the same phase ${ }^{2}$. In Quantum Mechanics, the probability finding particle is the square of amplitude at some point. Waves that superposition each other with same phase, will give highest amplitude among other path, so that's where particle goes.

It feels incomplete before we put in language of mathematics. Let me give you one more beautiful of physics (and mathematics of course, no doubt!), The Feynman Path Integral. Feyman Path 
Integral, or simply, path integral is a description of quantum theory that generalizes the action principle of classical mechanics. I will not give you the formulation of Path Integral here, seeing that i still not capable to do that. Briefly, Feynman said that the contribution of amplitude of a path is proportional to

$$
A \propto e^{-i S / \hbar}
$$

The path integral assigns to all these amplitdues equal weight but varying phase, or argument of the complex number. In macroscopic system, $S$ is very large compared to $\hbar$. So even a small (but still macroscopic) change in the action for such a particle will cause great changes in the phase. For such particles, many path amplitudes cancel each-other, making them unimportant for the particles way of motion. What we actually discover in reality is that only one certain path is followedthe classical trajectory $^{8}$.

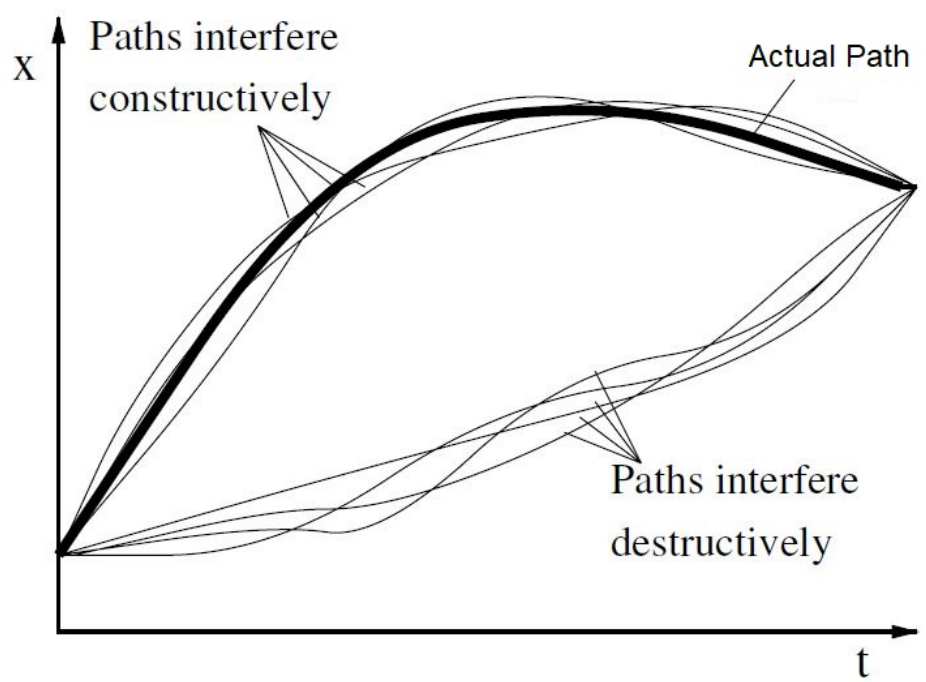

Figure 4. Emergence of the classical limit

For deeper understanding we see that the phase is not real function, but complex function. So we cannot see the inteference of this path happens in real space, shortly Euclidean Space. Because Quantum Mechanics lives in Hilbert Space!* Therefore, the overall probability amplitude of the system starting at $x_{a}$ at time $t_{a}$ and ending up at $x_{b}$ at time $t_{b}$ is given by the sum over all possible paths,

$$
P=\sum_{x} e^{-i S(x) / \hbar}
$$

If we draw graph of this function with real and imaginary parth separately, it gives

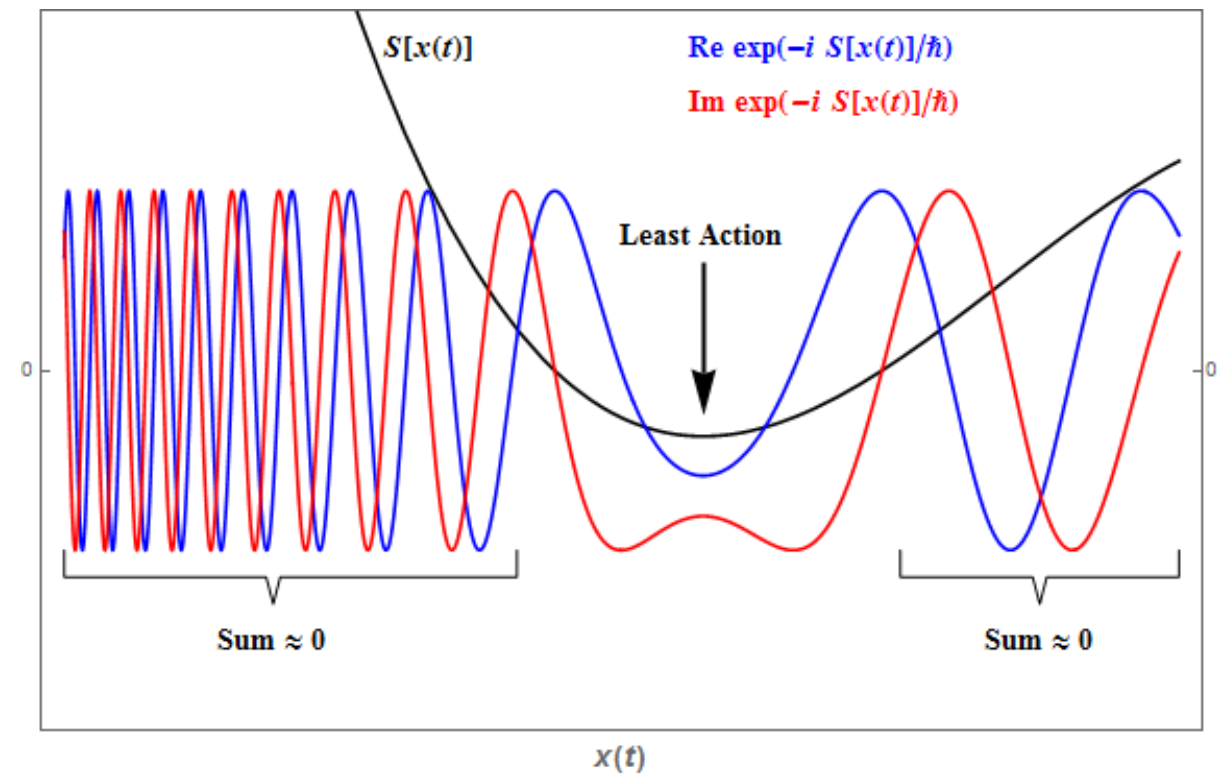

Figure.5

${ }^{*}$ One of my favorite quotation in physics, what a such astounding statement. 
The blue and red curves show respectively the real and imaginary parts of the complex exponential in the sum over paths above. You can see that when the action varies rapidly, this complex exponential function rapidly oscillates between -1 and 1 . When the action approaches a minimum, i.e. the point of least action (or any stationary point), the complex exponential function varies only slightly from the value at the stationary point ${ }^{4}$. The black curve is function of classical action, where the minimum point is non-zero sum over path of complex exponential (We integrate red and blue curves in this plot). Therefore, the paths that contribute the most to this sum are those near the path of least action, where the action varies more slowly.

In microscopic system, the action is typically of the order of $\hbar$. Hence it takes a comparably large change in the action to achieve a significant change in the phase $S(x)=\hbar$. In otherwords, for elementary or very light particles even paths that deviate much from the classical will be of importance. In this case one can no longer talk about the trajectory of the particle, but rather of a superposition of different paths ${ }^{8}$. That's what Quantum Mechanics is. Strange. In fact, that's what we really discovered in experiment, we actually proved that particle goes over all the path*

\section{References}

1. Baumann, Daniel.Least Action. Retrieved from www.damtp.cam.ac.uk/user/db275/LeastAction.pdf/. Accesed at October 282017.

2. Feynman, R., et al. (2005).The Feynman Lectures on Physics. US: Addison Wesley Pub. Co.,

3. Fitzpatrick, Richard.(2011). Hamilton's Equation. Retrieved from http://farside.ph.utexas.edu/ teaching/336k/Newtonhtml/node91.html. Accesed at October 302017.

4. Merthe, Daniel.(May 8 2017). Daniel Merthe's answer to What's the deeper meaning behind the principle of least action?. Quora. Retrieved From https://www.quora.com/Whats-thedeeper-meaning-behind-the-principle-of-least-action. Access at November 42017.

5. Liouville Equation. Retrieved from people.umass.edu/bvs/Lvll.pdf. Accesed at October 01 2017.

6. Roberts, Wayne. (2003). Principles of Nature, towards a new visual language. Australia: Self-Publishing

7. Thronton, Stephen T., Marion, Jerry B. 2004). Classical Dynamics of particles and systems 5th Edition. Brooks/Cole: United States of America.

8. Wikberg, E.(2006). Path Integrals in Quantum Mechanics. Stockholm University.

\footnotetext{
*For interested reader, See further in 'Double Slit' Experiment
} 